\title{
Current status of tertiary debulking surgery and prognosis after secondary debulking surgery for recurrent Müllerian epithelial cancer in Japan: a retrospective analysis of 164 patients (KCOG-G1402)
}

Tomoko Hirakawa ${ }^{1,2^{*}}$ (D, Takeo Minaguchi ${ }^{3}$, Yoshio Itani ${ }^{1,4}$, Yuka Kasamatsu $^{5}$, Saki Murase ${ }^{6}$, Shoko Sakurada ${ }^{7}$ Hiroaki Nagano ${ }^{1,8}$, Kazuhiro Takehara9 ${ }^{9}$, Tomohiko Tsuruta ${ }^{1,10}$, Atsushi Arakawa ${ }^{1,11}$, Kouichiro Kawano ${ }^{12}$, Hiroshi Tsubamoto ${ }^{1,13}$, Takashi Ushiwaka ${ }^{1,14}$, Taisuke Mori ${ }^{1,15}$, Kana Iwai ${ }^{16}$, Motoaki Saito ${ }^{17}$, Hiroyuki Morisawa ${ }^{18}$, Fumitaka Saito ${ }^{19}$, Kenta Yoshida ${ }^{1,20}$, Masanori Kaneuchi ${ }^{21}$, Hiroki Sato ${ }^{2}$, Kimihiko Ito ${ }^{1,10}$ and Kaei Nasu ${ }^{1,2}$

\begin{abstract}
Background: This study aimed to evaluate the current status of secondary debulking surgery (SDS) and tertiary debulking surgery (TDS; performed for recurrence after SDS) and to assess the overall survival after recurrence of Müllerian epithelial cancer in Japan. We also evaluated the data of patients who underwent a fourth debulking surgery (i.e., quaternary debulking surgery (QDS)).
\end{abstract}

Methods: We conducted a retrospective study of 164 patients with recurrent Müllerian epithelial cancers (i.e., ovarian, tubal, and peritoneal cancers). The SDS was performed between January 2000 and September 2014 in 20 Japanese hospitals. Clinicopathological data were collected and analyzed.

Results: Of the 164 patients, 66 patients did not have a recurrence or died after SDS. Ninety-eight patients had a recurrence after SDS. Forty-three of the 98 patients underwent TDS; 55 of the 98 patients did not undergo TDS and were classified into the non-TDS group. The overall survival (OS) after SDS was significantly better in the TDS group than in the non-TDS group. The median OS after SDS was 123 and 42 months in the TDS group and non-TDS group, respectively. Of the 43 patients who received TDS, 11 patients were further treated with QDS. The median OS after SDS was 123 months for patients who underwent QDS.

Conclusions: This multicenter study on the prognosis of post-SDS is apparently the first report on QDS in Japan. Patients undergoing TDS have a good prognosis, compared to patients in the non-TDS group. Novel drugs are being evaluated; however, debulking surgery remains a necessary treatment for recurrence.

Keywords: Müllerian epithelial cancer, Recurrence, Secondary debulking surgery, Tertiary debulking surgery, Quaternary debulking surgery

\footnotetext{
* Correspondence: tomokoh@oita-u.ac.jp

${ }^{1}$ Kansai Clinical Oncology Group (KCOG), 5-30 Tennoji, Osaka 543-8555, Japan

${ }^{2}$ Department of Obstetrics and Gynecology, Faculty of Medicine, Oita

University, 1-1 Idaigaoka, Hasama-machi, Yufu-shi, Oita 879-5593, Japan

Full list of author information is available at the end of the article
} 


\section{Background}

The treatment of recurrent ovarian cancer consists mainly of chemotherapy. However, secondary debulking surgery (SDS; also known as secondary cytoreductive surgery) is an effective option if the patients are selected carefully [1-4]. There are very few reports on Japanese patients treated with SDS, although there is one previous report on 44 Japanese patients [5]. Secondary debulking surgery seems to be effective if complete resection has been achieved. The AGO DESKTOP OVAR Trial (DESKTOP I trial) showed that only complete surgery was associated with prolonged survival in patients with recurrent ovarian cancer [6]. Moreover, the trial presented a predictive score for the resectability of recurrent ovarian cancer. This score model was based on the performance status, the presence of ascites, and the outcome of the primary surgery $[6,7]$. The DESKTOP II trial was subsequently the first prospective multicenter trial that successfully validated a clinical score that could be used to predict complete resection [8].

Surgery after SDS (i.e., tertiary debulking surgery (TDS)) is performed in highly selected cases and is rarely reported [9-13]. Moreover, reports of a fourth debulking surgery (i.e., quaternary debulking surgery (QDS)) are even rarer [14-17]. If the efficacy of SDS can be proven, the effectiveness of TDS or additional debulking surgeries should be studied.

With this in mind, the aims of this study were to retrospectively analyze the data of patients with recurrent Müllerian epithelial cancers (i.e., ovarian, tubal, and peritoneal cancers) and to assess the prognostic factors and the current status of recurrence after SDS in Japan.

\section{Methods}

We designed a multicenter retrospective study, which included 164 patients with recurrent Müllerian epithelial cancer who were enrolled from 20 hospitals in Japan. The Institutional Review Boards of each hospital approved this study. The patient selection criteria were as follows: a complete response was achieved by the primary treatment, patients were histologically diagnosed with Müllerian epithelial cancers (i.e., ovarian, tubal, and peritoneal cancers), and SDS was performed between January 2000 and September 2014. The performance status of the patients was estimated to be 0 or 1 , based on the Eastern Cooperative Oncology Group Performance Status (ECOG-PS) criteria. The exclusion criteria were as follows: a history of other cancer, patients who did not achieve complete response by SDS or subsequent chemotherapy, and patients who underwent palliative surgery at first recurrence (therefore, it was not regarded as SDS). The collected data about the initial treatment included age at the initial diagnosis, clinical and pathological stage (i.e., International Federation of Gynecology and Obstetrics (FIGO) stage), histology, serum cancer antigen-125 (CA125) level, and presence of residual tumor $(0,<10$, or $\geq 10 \mathrm{~mm})$ at the primary debulking surgery (PDS). The data collected at recurrence included age, platinum-free interval, serum CA125 level, presence of peritonitis carcinomatosa, and presence of residual tumors at $\operatorname{SDS}(0,<10$, or $\geq 10 \mathrm{~mm})$. The collected data at the TDS (i.e., recurrence after SDS) were age, serum CA125 level, presence of peritonitis carcinomatosa, site of recurrence, and number of recurrence. Survival time after SDS and data regarding TDS and QDS were also collected. The absence of residual tumor $(0 \mathrm{~mm})$ was defined as a complete surgery. The TDS group consisted of patients who underwent debulking surgery for recurrence after SDS. Patients undergoing a fourth or fifth debulking surgery were also included in the TDS group. The non-TDS group consisted of patients who did not undergo a TDS; these patients had not undergone surgery for recurrence after the SDS.

The primary objective was to compare the overall survival (OS) in the TDS group with that in the non-TDS group. The OS was the interval between the date of the SDS and death. Most studies define OS as the survival time from the initial diagnosis. However, in this study, all patients underwent a SDS. The time between the primary treatment and recurrence ranged widely among them; therefore, the definition of OS as the survival time between the date of SDS and death was appropriate.

The secondary objective was to identify the characteristics of the TDS group and the non-TDS group. The platinum-free interval was the interval between the date of the last infusion of platinum for the primary treatment and the date of recurrence. Platinum resistance was defined as recurrence within 6 months after the last platinum treatment. Platinum partial sensitivity was defined as recurrence between 6 and 12 months after platinum treatment. Platinum sensitivity was defined as recurrence after 12 months.

The differences in the background characteristics of the patients were analyzed using the chi-square test. The survival data were analyzed using the Kaplan-Meier method and log-rank test. For all analyses, Statistical Package for Social Science software (IBM SPSS Statistics 24; IBM, Armonk, NY) was used, and $P<0.05$ was considered statistically significant.

\section{Results}

Figure 1 shows the inclusion criteria for data collection in this study. One hundred sixty-four patients underwent SDS in 20 hospitals. Sixty-six patients did not have a recurrence or died after SDS. Ninety-eight patients had a recurrence after SDS. Forty-three of the 98 patients were classified into the TDS group. Tertiary debulking surgery was performed in 15 hospitals. Among the TDS group, 11 


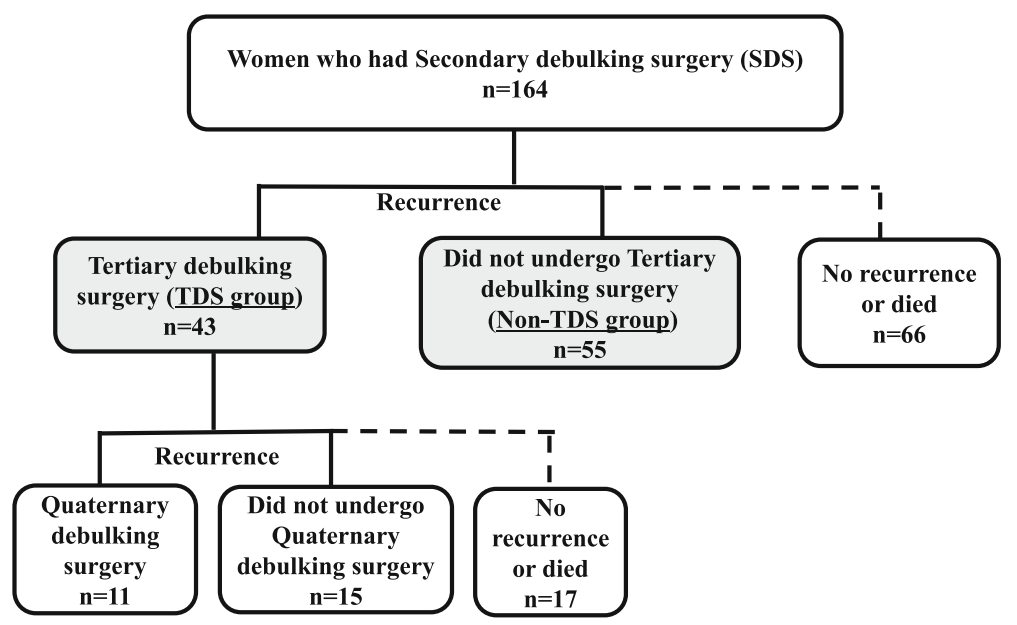

Fig. 1 The inclusion criteria of patients in this study

(25.6\%) patients underwent QDS, 15 (34.9\%) patients did not undergo QDS, and 17 (39.5\%) patients did not have a recurrence or died after TDS.

Fifty-five of the 98 patients did not undergo TDS when they had a recurrence after SDS, and they were classified into the non-TDS group. The non-TDS patients were treated with chemotherapy or the best supportive care.

Table 1 shows the main characteristics of the patients in two groups at the time of initial diagnosis, SDS, and recurrence after SDS. The median ages of the patients at initial diagnosis were 56.9 years (range, 35-74 years) and 54.2 (range, 27-77 years) in the TDS and non-TDS groups, respectively. Forty-two (97.7\%) patients in the TDS group and 51 (92.7\%) patients in the non-TDS group were diagnosed with ovarian cancer. No cases of tubal cancer were included in the TDS group. There were only two (3.6\%) cases of tubal cancer in the non-TDS group. One $(2.3 \%)$ patient in the TDS group and two (3.6\%) patients in the non-TDS group had peritoneal cancer. Serous cystadenocarcinoma was the most common histological type (22 [51.2\%] cases in the TDS group and 36 [65.5\%] cases in the non-TDS group). Complete surgery at PDS was performed in $34(79.1 \%)$ patients in the TDS group and 33 $(60.0 \%)$ patients in the non-TDS group. Complete surgery at SDS was performed in $38(88.4 \%)$ patients in the TDS group and $44(80.0 \%)$ patients in the non-TDS group.

The median ages at TDS recurrence (i.e., recurrence after SDS) were 61.5 years (range, $40-78$ years) and 58.6 years (range, $33-80$ years) in the TDS and non-TDS groups, respectively. The CA125 levels at TDS recurrence (i.e., recurrence after SDS) significantly differed between the two groups $(P=0.040)$. Peritonitis carcinomatosa was significantly different in the non-TDS patients $(P=0.042)$. With regard to the sites of recurrence, more patients showed recurrence in the peritoneal cavity in the nonTDS group than in the TDS group $(P=0.009)$. In the TDS and non-TDS groups, peritoneal recurrence occurred in $28(65.1 \%)$ patients and $48(87.3 \%)$ patients, respectively, while extra-peritoneal recurrence occurred in 15 (34.9\%) patients and seven (12.7\%) patients, respectively. Among cases of extra-peritoneal recurrence in the TDS group, five (33.3\%) cases occurred in the lung; five (33.3\%) cases occurred in the brain; four (26.7\%) cases occurred in the inguinal lymph nodes; and one $(6.7 \%)$ case occurred in the axilla. In the non-TDS group, the extra-peritoneal recurrence site was the lung in three $(42.9 \%)$ cases, the mediastinal space in three $(42.9 \%)$ cases, and the bone in one $(14.3 \%)$ case. With regard to the number of recurrence sites, more patients presented with single-site recurrence in the TDS group than in the non-TDS group $(P=0.013)$. Solitary recurrence occurred in $24(55.8 \%)$ TDS patients and 17 (30.9\%) non-TDS patients, and multiple recurrences occurred in 19 (44.2\%) TDS patients and 38 (69.1\%) non-TDS patients.

Among the 164 patients included in this study, 43 patients underwent TDS. The OS after SDS was significantly better in the TDS group, compared to the non-TDS group $(P=0.001$; Fig. 2). The median OS after SDS was 123 months and 42 months in the TDS group and nonTDS group, respectively.

Our study included 11 patients who underwent QDS (Table 2). Moreover, two of the 11 QDS patients underwent a fifth debulking surgery. Eight of the 11 patients were alive at the conclusion of the study. The median age of the 11 patients at QDS was 57 years (range, 5265 years), and the median OS after SDS was 123 months (range, 43-138 months; Table 2).

\section{Discussion}

This study aimed to clarify the current status of SDS and TDS in Japan because few patients have undergone SDS or TDS in Japan and the current status of SDS and 
Table 1 Clinical characteristics of the tertiary debulking surgery (TDS) group and non-TDS group

\begin{tabular}{|c|c|c|c|c|c|c|c|}
\hline Characteristics & $\operatorname{TDS}(n=43)$ & Non-TDS $(n=55)$ & $P$ value & Missing & $1(2.3 \%)$ & $3(5.5 \%)$ & \\
\hline \multicolumn{4}{|l|}{ Primary } & \multicolumn{4}{|l|}{ Residual disease at SDS, mm } \\
\hline Age, median (range), years & $56.9(35-74)$ & $54.2(27-77)$ & \multirow{3}{*}{0.432} & 0 & $38(88.4 \%)$ & $44(80.0 \%)$ & \multirow[t]{5}{*}{0.092} \\
\hline$<60$ & $24(55.8 \%)$ & $35(63.6 \%)$ & & $0-10$ & $1(2.3 \%)$ & $8(14.5 \%)$ & \\
\hline$\geqq 60$ & $19(44.2 \%)$ & $20(36.4 \%)$ & & $\geqq 10$ & $2(4.7 \%)$ & $1(1.8 \%)$ & \\
\hline Cancer & & & & Missing & $2(4.7 \%)$ & $2(3.6 \%)$ & \\
\hline Ovary & $42(97.7 \%)$ & $51(92.7 \%)$ & \multirow[t]{3}{*}{0.415} & Recurrence at TDS & & & \\
\hline Fallopian tube & $0(0 \%)$ & $2(3.6 \%)$ & & Age, median (range), years & $61.5(40-78)$ & $58.6(33-80)$ & \multirow{3}{*}{0.262} \\
\hline Peritoneum & $1(2.3 \%)$ & $2(3.6 \%)$ & & $<60$ & $17(39.5 \%)$ & $28(50.9 \%)$ & \\
\hline FIGO stage & & & & $\geqq 60$ & $26(60.5 \%)$ & $27(49.1 \%)$ & \\
\hline ।, II & $10(23.3 \%)$ & $13(23.6 \%)$ & \multirow[t]{2}{*}{0.965} & CA $125(\mathrm{U} / \mathrm{ml})$ & & & \\
\hline III, IV & $33(76.7 \%)$ & $42(76.4 \%)$ & & $<100$ & $31(72.1 \%)$ & $30(54.5 \%)$ & \multirow[t]{2}{*}{0.040} \\
\hline Histology & & & & $\geqq 100$ & $7(16.3 \%)$ & $19(34.5 \%)$ & \\
\hline Serous & $22(51.2 \%)$ & $36(65.5 \%)$ & \multirow[t]{5}{*}{0.333} & Missing & $5(11.6 \%)$ & $6(10.9 \%)$ & \\
\hline Endometrioid & $6(14.0 \%)$ & $4(7.3 \%)$ & & Peritonitis carcinomatosa & & & \\
\hline Clear cell & $10(23.3 \%)$ & $6(10.9 \%)$ & & No & $40(93.0 \%)$ & $46(83.6 \%)$ & \multirow[t]{3}{*}{0.042} \\
\hline Mucinous & $2(4.7 \%)$ & $3(5.5 \%)$ & & Yes & $0(0 \%)$ & $5(9.1 \%)$ & \\
\hline Others & $3(7.0 \%)$ & $6(10.9 \%)$ & & Missing & $3(7.0 \%)$ & $4(7.3 \%)$ & \\
\hline \multicolumn{4}{|l|}{ CA $125(\mathrm{U} / \mathrm{ml})$} & \multicolumn{4}{|l|}{ Sites of recurrence } \\
\hline$<100$ & $10(23.3 \%)$ & 7 (12.7\%) & \multirow[t]{3}{*}{0.086} & Peritoneal & $28(65.1 \%)$ & $48(87.3 \%)$ & \multirow[t]{3}{*}{0.009} \\
\hline$\geqq 100$ & $21(48.8 \%)$ & $38(69.1 \%)$ & & Extra-peritoneal & $15(34.9 \%)$ & $7(12.7 \%)$ & \\
\hline Missing & $12(27.9 \%)$ & $10(18.2 \%)$ & & Number of recurrence & & & \\
\hline \multicolumn{2}{|l|}{ Residual disease at PDS, mm } & & & Single & $24(55.8 \%)$ & 17 (30.9\%) & \multirow[t]{2}{*}{0.013} \\
\hline 0 & $34(79.1 \%)$ & $33(60.0 \%)$ & 0.035 & Multiple & $19(44.2 \%)$ & $38(69.1 \%)$ & \\
\hline
\end{tabular}

Table 1 Clinical characteristics of the tertiary debulking surgery (TDS) group and non-TDS group (Continued)

TDS is unknown. We found no significant difference in the characteristics between the TDS and non-TDS groups at the primary treatment and at the first recurrence, except for residual disease at PDS. However, a significant difference was noted between the TDS and non-TDS groups in the survival time from the SDS $(P<$ 0.001 , Fig. 2). It has been previously demonstrated that SDS shows an advantage in terms of longer OS for highly selected patients $[18,19]$; however, the predictors of survival in these patients remain unclear.

It has been reported that ascites $(<500 \mathrm{ml})$ is a prognostic factor and predictor of complete surgery $[6,7]$. In this study, it was difficult to collect data concerning ascites because amount of ascites was not measured in most patients. Therefore, we evaluated ascites by using the presence of peritonitis carcinomatosa. In the present study, only three (7.0\%) patients and four $(7.3 \%)$ patients relapsed with carcinomatous peritonitis in the TDS and non-TDS groups at SDS, respectively. It is possible that the patients with ascites were treated largely by chemotherapy instead of SDS, although this study has no data on the non-SDS patients (i.e., patients who did not receive 


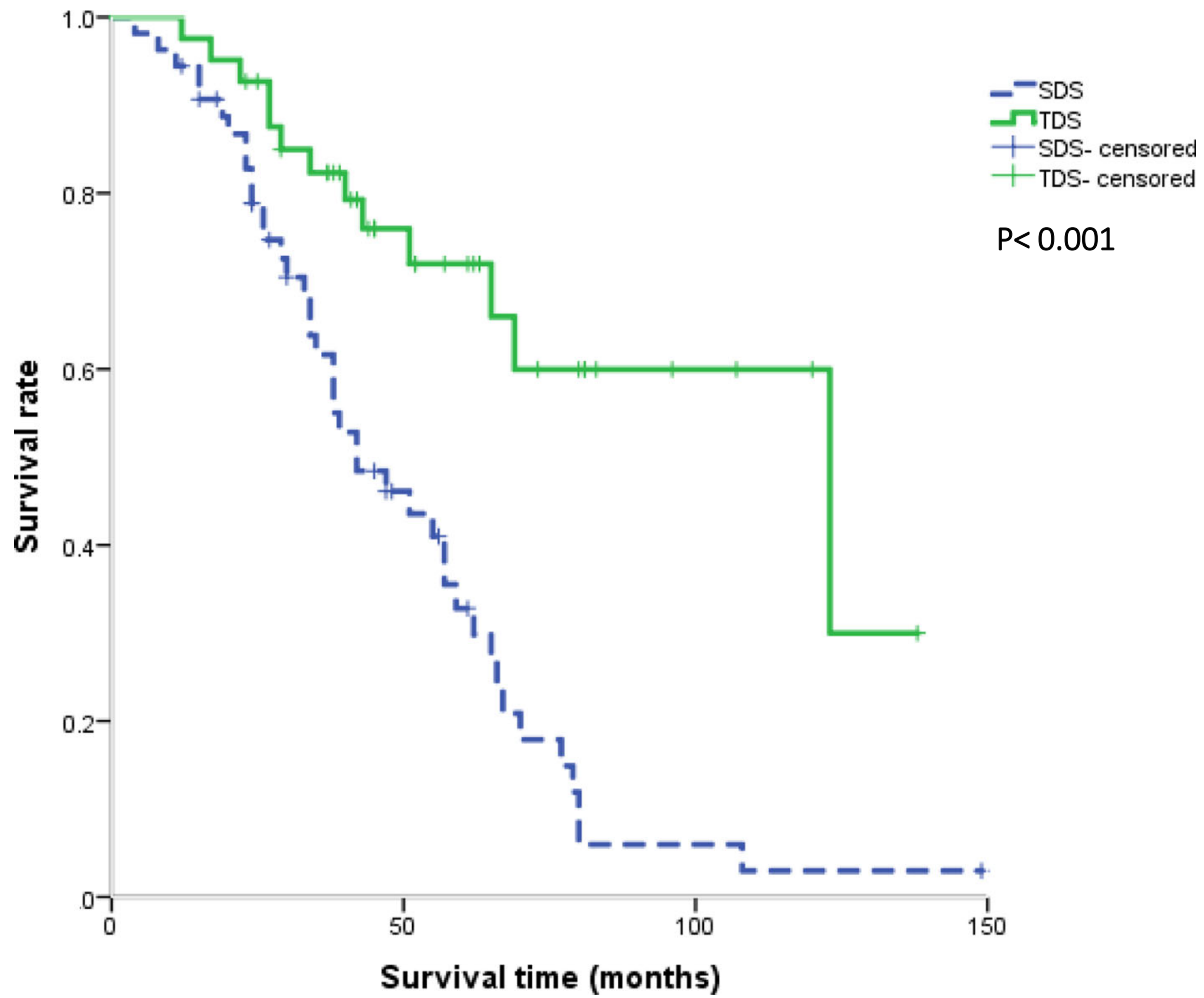

Fig. 2 Kaplan-Meier plot of survival rates after SDS. The survival rate was significantly better in the TDS group than in the non-TDS group $(P<$ 0.001). The median OS after SDS was 123 months in the TDS group and 42 months in the non-TDS group. SDS secondary debulking surgery, TDS tertiary debulking surgery, OS overall survival

Table 2 Patients characteristics at the time of quaternary debulking surgery (QDS)

\begin{tabular}{|c|c|c|c|c|c|c|c|c|}
\hline Author & Year & Number & FIGO stage $(n)$ & $\mathrm{Age}^{\mathrm{a}}$ & Residual disease $(n)$ & Median CA125 (U/ml) & Chemotherapy $(n)$ & Median OS $(m)^{b}$ \\
\hline \multirow[t]{4}{*}{ Shih et al. } & 2010 & 15 & I (1) & 54.1 & 0 mm (10) & NA & Yes (9) & $34.8,10.1$ (from TDS) $^{c}$ \\
\hline & & & $\|(2)$ & & $>0$ mm (5) & & No (6) & \\
\hline & & & III (9) & & & & & \\
\hline & & & IV (1) & & & & & \\
\hline \multirow[t]{4}{*}{ Fotopoulou et al. } & 2013 & 49 & I (6) & 57 & 0 mm (16) & 736 & Yes (18) & 23.05 (from QDS) \\
\hline & & & $\|(6)$ & & $>0$ mm (33) & & No (31) & \\
\hline & & & III (33) & & & & & \\
\hline & & & IV (1) & & & & & \\
\hline \multirow[t]{4}{*}{ Bacarubasa et al. } & 2015 & 20 & I (4) & 54.3 & 0 mm (7) & NA & NA & 16 (from QDS) \\
\hline & & & $\|(5)$ & & $>0$ mm (13) & & & \\
\hline & & & III (11) & & & & & \\
\hline & & & IV (0) & & & & & \\
\hline \multirow[t]{4}{*}{ Hirakawa et al. } & 2017 & 11 & I (0) & 57 & 0 mm (7) & 11.2 (6 cases) & Yes (4) & 123 (from SDS) \\
\hline & & & $\|(1)$ & & $>0$ mm (2) & & No $(7)$ & \\
\hline & & & III (7) & & Missing (2) & & & \\
\hline & & & IV (3) & & & & & \\
\hline
\end{tabular}

${ }^{\mathrm{b}}$ The values are the median overall survival (OS)

${ }^{\mathrm{c}}$ For residual disease $\leq 10 \mathrm{~mm}$, the median OS was 34.8 months; for residual disease $>10 \mathrm{~mm}$, the median OS was 10.1 months 
SDS at first recurrence). At the time of TDS (i.e., recurrence after SDS), more patients in the non-TDS group had a recurrence in the peritoneal cavity, compared to the TDS group $(P=0.009)$. Moreover, more patients showed single-site recurrence in the TDS group than in the nonTDS group $(P=0.013)$. Many cases of peritoneal cavity recurrence involved wide peritoneal dissemination. These patients may have selected chemotherapy instead of TDS.

In the TDS group, 11 patients underwent QDS and two of these 11 patients underwent a fifth debulking surgery. Ten $(90.9 \%)$ of the 11 patients with FIGO stage III and IV cancers were in the QDS group. This study included higher ratio of advanced stage cancer patients, compared to other reports on patients with QDS (Table 2). Based on these results, we believe that this is a valuable study to clarify the current status of TDS or additional debulking surgeries.

There are several limitations of this study. One of two main limitations is the retrospective nature of the study. The second main limitation is the small sample size per year and the small number of institutions. Among the 164 patients over 14 years, only approximately 12 patients underwent SDS per year across 20 institutions. We assumed that very few Japanese patients were treated with SDS. Therefore, we collected data from many hospitals in Japan. Despite the smaller sample size per institution, this study provides valuable data concerning TDS and QDS.

A third limitation is the potential for selection bias, such as similar tumor sizes and number of relapse sites. This bias might influence the presence of residual tumor after debulking surgery and/or the survival time. In future studies, selection bias in evaluating the efficacy of SDS and TDS needs to be overcome. We could not rule out the possibility that only highly selected patients were operated on in the present study; nevertheless, this is a valuable study on the current status of SDS and TDS. To the best of our knowledge, this is the first report on TDS and QDS in Japan.

\section{Conclusions}

In conclusion, several novel agents such as monoclonal antibodies are being evaluated, although debulking surgery remains an important treatment option for multiple relapses. The present study demonstrated that, at second recurrence, patients who were treated with TDS showed a favorable OS, compared to patients treated with chemotherapy or best supportive care. Further reports of patients treated with SDS or additional debulking surgery need to be accumulated to evaluate treatment results and confirm our findings.

\section{Abbreviations}

OS: Overall survival; QDS: Quaternary debulking surgery; SDS: Secondary debulking surgery; TDS: Tertiary debulking surgery

Acknowledgements

Not applicable
Funding

This study was not funded by an outside source.

Availability of data and materials

The authors respect the patients' right to privacy. We presented all important information in this published article. Please contact the corresponding author if the data are needed.

\section{Authors' contributions}

$\mathrm{TH}, \mathrm{KI}$, and $\mathrm{KN}$ designed this protocol. TH, TM, YI, YK, SM, SS, HN, KT, TT, AA KK, HT, UT, TM, KI, MS, HM, FS, KY, and MK collected the data. TH and HS analyzed the data. TH wrote this manuscript. All authors read and approved the final manuscript.

\section{Ethics approval and consent to participate}

In this retrospective study, clinical data were anonymized and this study was approved by the Institutional Review Board of every hospital.

\section{Consent for publication}

Not applicable.

\section{Competing interests}

The authors declare that they have no competing interests.

\section{Publisher's Note}

Springer Nature remains neutral with regard to jurisdictional claims in published maps and institutional affiliations.

\section{Author details}

${ }^{1}$ Kansai Clinical Oncology Group (KCOG), 5-30 Tennoji, Osaka 543-8555, Japan. ${ }^{2}$ Department of Obstetrics and Gynecology, Faculty of Medicine, Oita University, 1-1 Idaigaoka, Hasama-machi, Yufu-shi, Oita 879-5593, Japan. ${ }^{3}$ Department of Obstetrics and Gynecology, Faculty of Medicine, University of Tsukuba, Tsukuba, Ibaraki 305-8576, Japan. ${ }^{4}$ Department of Obstetrics and Gynecology, Nara Prefecture General Medical Center, Nara 631-0846, Japan. ${ }^{5}$ Division of Gynecology, Shizuoka Cancer Center Hospital, Suntou, Shizuoka 411-8777, Japan. 'Department of Obstetrics and Gynecology, Gifu University, Gifu 501-1194, Japan. ${ }^{7}$ Department of Obstetrics and Gynecology, Tohoku University School of Medicine, Sendai, Miyagi 980-8574, Japan. ${ }^{8}$ Department of Obstetrics and Gynecology, Tokyo Women s Medical University Medical Center East, Arakawa, Tokyo 116-8567, Japan. ${ }^{9}$ Department of Gynecologic Oncology, Shikoku Cancer Center, Matsuyama, Ehime 791-0280, Japan. ${ }^{10}$ Department of Obstetrics and Gynecology, Kansai Rosai Hospital, Amagasaki, Hyogo 660-8511, Japan. ${ }^{11}$ Department of Obstetrics and Gynecology, Nagoya City University Graduate School of Medical Sciences, Nagoya, Aichi 467-8602, Japan. ${ }^{12}$ Department of Obstetrics and Gynecology, Kurume University School of Medicine, Kurume, Fukuoka 830-0011, Japan.

${ }^{13}$ Department of Obstetrics and Gynecology, Hyogo College of Medicine, Nishinomiya, Hyogo 663-8501, Japan. ${ }^{14}$ Department of Obstetrics and Gynecology, Kochi Medical School, Nankoku, Kochi 783-8505, Japan.

${ }^{15}$ Department of Obstetrics and Gynecology, Kyoto Prefectural University of Medicine, Kyoto 602-8566, Japan. ${ }^{16}$ Department of Obstetrics and Gynecology, Nara Medical University, Kashihara, Nara 634-8521, Japan. ${ }^{17}$ Department of Obstetrics and Gynecology, Jikei University School of Medicine, Minato-ku, Tokyo 105-8471, Japan. ${ }^{18}$ Department of Obstetrics and Gynecology, Jichi Medical University, Shimotsuke, Tochigi 329-0498, Japan.

${ }^{19}$ Department of Obstetrics and Gynecology, Faculty of Life Science, Kumamoto University, Kumamoto 860-8556, Japan. ${ }^{20}$ Department of Obstetrics and Gynecology, Mie University School of Medicine, Tsu, Mie 514-8507, Japan. ${ }^{21}$ Department of Obstetrics and Gynecology, Nagasaki University, Nagasaki 852-8501, Japan.

Received: 6 November 2016 Accepted: 3 July 2017 Published online: 17 July 2017

\section{References}

1. Bristow RE, Puri I, Chi DS. Cytoreductive surgery for recurrent ovarian cancer: a meta-analysis. Gynecol Oncol. 2009;112:265-74.

2. Nasu K, Kai K, Hirakawa T, Nishida M, Matsumoto H, Kawano Y, et al. Retrospective analysis of outcomes of secondary debulking surgery for 
recurrent epithelial ovarian cancer with favorable prognostic factors. J Obstet Gynaecol Res. 2014;40:791-6.

3. Laas E, Luyckx M, De Cuypere M, Selle F, Daraï E, Querleu D, et al. Secondary complete cytoreduction in recurrent ovarian cancer: benefit of optimal patient selection using scoring system. Int J Gynecol Cancer. 2014;24:238-46.

4. Zang RY, Li ZT, Tang J, Cheng X, Cai SM, Zhang ZY, et al. Secondary cytoreductive surgery for patients with relapsed epithelial ovarian carcinoma: who benefits? Cancer. 2004;100:1152-61.

5. Onda T, Yoshikawa H, Yasugi T, Yamada M, Matsumoto K, Taketani Y, et al. Secondary cytoreductive surgery for recurrent epithelial ovarian carcinoma: proposal for patients selection. Br J Cancer. 2005;92:1026-32.

6. Harter P, du Bois A, Hahmann M, Hasenburg A, Burges A, Loibl S, Arbeitsgemeinschaft Gynaekologische Onkologie Ovarian Committee, AGO Ovarian Cancer Study Group, et al. Surgery in recurrent ovarian cancer: the Arbeitsgemeinschaft Gynaekologische Onkologie (AGO) DESKTOP OVAR trial. Ann Surg Oncol. 2006;13:1702-10.

7. Harter P, Hahmann M, Lueck HJ, Poelcher M, Wimberger P, Ortmann O, et al. Surgery for recurrent ovarian cancer: role of peritoneal carcinomatosis: exploratory analysis of the DESKTOP I trial about risk factors, surgical implications, and prognostic value of peritoneal carcinomatosis. Ann Surg Oncol. 2009;16:1324-30.

8. Harter P, Sehouli J, Reuss A, Hasenburg A, Scambia G, Cibula D, et al. Prospective validation study of a predictive score for operability of recurrent ovarian cancer: the Multicenter Intergroup Study DESKTOP II. A project of the AGO Kommission OVAR, AGO Study Group, NOGGO, AGO-Austria, and MITO. Int J Gynecological cancer. 2011;21:289-95.

9. Dogan NU, Schneider A, Chiantera V, Dogan S, Dursun P. Tertiary cytoreduction in the setting of recurrent ovarian cancer (Review). Oncol Lett. 2013;6:642-7.

10. Tang J, Liu DL, Shu S, Tian WJ, Liu Y, Zang RY. Outcomes and patterns of secondary relapse in platinum-sensitive ovarian cancer: implications for tertiary cytoreductive surgery. Eur I Surg Oncol. 2013;39:786-91.

11. Hızlı D, Boran N, Yılmaz S, Turan T, Alıınbaş SK, Celik B, et al. Best predictors of survival outcome after tertiary cytoreduction in patients with recurrent platinum-sensitive epithelial ovarian cancer. Eur J Obstet Gynecol Reprod Biol. 2012;163:71-5.

12. Shih KK, Chi DS, Barakat RR, Leitao Jr MM. Tertiary cytoreduction in patients with recurrent epithelial ovarian, fallopian tube, or primary peritoneal cancer: an updated series. Gynecol Oncol. 2010;117:330-5.

13. Gultekin M, Velipaşaoğlu M, Aksan G, Dursun P, Dogan NU, Yuce K, et al. A third evaluation of tertiary cytoreduction. J Surg Oncol. 2008;98:530-4.

14. Shih KK, Chi DS, Barakat RR, Leitao Jr MM, et al. Beyond tertiary cytoreduction in patients with recurrent epithelial ovarian, fallopian tube, or primary peritoneal cancer. Gynecol Oncol. 2010;116:364-9.

15. Bacalbaşa N, Balescu I, Dima S, Brasoveanu V, Popescu I. The role of quaternary cytoreduction in recurrent epithelial ovarian cancer: a singlecenter experience. Anticancer Res. 2015;35:3519-23.

16. Fotopoulou C, Savvatis K, Kosian P, Braicu IE, Papanikolaou G, Pietzner K et al. Quaternary cytoreductive surgery in ovarian cancer: does surgical effort still matter? Br J Cancer. 2013;108:32-8.

17. Shih KK, Chi DS. Maximal cytoreductive effort in epithelial ovarian cancer surgery. J Gynecol Oncol. 2010;21:75-80.

18. da Costa AA, Valadares CV, Mantoan H, Saito A, Salvadori MM, Guimarães $A P$, et al. The value of secondary cytoreductive surgery in recurrent ovarian cancer and application of a prognostic score. Int J Gynecol Cancer. 2016;26: 449-55.

19. Chuang CM, Chou YJ, Yen MS, Chao KC, Twu NF, Wu HH, et al. The role of secondary cytoreductive surgery in patients with recurrent epithelial ovarian, tubal, and peritoneal cancers: a comparative effectiveness analysis. Oncologist. 2012;17:847-55.

\section{Submit your next manuscript to BioMed Central and we will help you at every step:}

- We accept pre-submission inquiries

- Our selector tool helps you to find the most relevant journal

- We provide round the clock customer support

- Convenient online submission

- Thorough peer review

- Inclusion in PubMed and all major indexing services

- Maximum visibility for your research

Submit your manuscript at www.biomedcentral.com/submit
Biomed Central 